\title{
空气氛围下可见光诱导的硫酚氧化脱氢偶联
}

\author{
陈幸周璇姬小趁黄华文* \\ (湘潭大学化学学院 湖南湘潭 411105)
}

\begin{abstract}
摘要 $\alpha$-硫醚化羰基化合物广泛存在于自然界和各行业领域，是合成杂环的重要中间体，传统的羰基硫醚合成方法通 常需要将底物预功能化, 将其从亲核试剂转化为亲电试剂, 合成方法繁琐复杂, 因此探索一种新型的羰基化合物的硫 醚化反应非常重要. 开发了一种在可见光条件下，利用酮类化合物和硫酚的氧化脱氢偶联反应合成硫醚化合物的方法. 在光催化体系下, 无需任何金属, 该反应使用廉价易得的空气作为氧化剂, 成功实现高原子经济性和广泛的底物适用 范围, 为 $\mathrm{C}-\mathrm{S}$ 键的直接构建提供了更为简便的方法.
\end{abstract}

关键词 可见光诱导; $\mathrm{C}-\mathrm{S}$ 键构建; 脱氢偶联; 硫梄

\section{Visible Light-Induced Aerobic Oxidative Dehydrogenative Coupling of Thiophenols}

\author{
Chen, Xing Zhou, Xuan Ji, Xiaochen Huang, Huawen* \\ (College of Chemistry, Xiangtan University, Xiangtan, Hunan 411105)
}

\begin{abstract}
Thioetherified carbonyl compounds widely exist in nature and various industries. They are important intermediates for the synthesis of heterocycles. The traditional synthesis methods of carbonyl thioethers usually need the prefunctionalization of substrates to convert them from nucleophiles to electrophiles. The synthesis methods are cumbersome and complex. Therefore, it is very important to explore a new thioetherification reaction of carbonyl compounds. The synthesis of thioether compounds by oxidative dehydrogenation coupling reaction of ketones and thiophenols under visible light was developed. In the photocatalytic system, without any metal, the reaction successfully realizes high atomic economy and wide substrate application range using cheap and easily available air as oxidant, and provides a simpler method for the direct construction of $\mathrm{C}-\mathrm{S}$ bond.

Keywords photocatalysis; $\mathrm{C}-\mathrm{S}$ bond construction; dehydrogenation coupling; thioether
\end{abstract}

硫醚化合物具有显著的生物活性，广泛存在于与生 活息息相关的医药、天然产物和功能材料等领域 ${ }^{[1]}$. 因 此, $\mathrm{C}-\mathrm{S}$ 键形成反应的发展在有机合成中受到了广泛 关注 ${ }^{[2]}$. 传统的制备方法是通过羰基化合物与硫醇或硫 酚等硫源的亲核取代反应 ${ }^{[3]}$, 这些方法大多有着成本高 及对反应环境敏感等局限性, 而之后报道的借助 $\mathrm{C}-\mathrm{H} /$ $\mathrm{S}-\mathrm{H}$ 的氧化偶联反应直接构建 $\mathrm{C}-\mathrm{S}$ 键则在反应局限 性和产率方面显示出明显优势. 铜、铁和镍等过渡金属 催化体系 ${ }^{[4]}$ 偶联形成 $\mathrm{C}-\mathrm{S}$ 键的反应也得到了迅猛的发 展. 2004 年, Ranu 课题组 ${ }^{[5]}$ 研究了二硫化物与卤代烷基 或酰基缩合生成硫醚化合物的反应，在该体系中，碘化
铟被用来催化 $\mathrm{S}-\mathrm{S}$ 键的断裂, 该反应适用性强, 操作 简单，反应速度快，分离产率高.

随着绿色有机合成的提出, 绿色环保的电催化和光 催化体系逐渐引起了科研工作者的浓厚兴趣 ${ }^{[6]}$. 由于不 需要底物的预功能化, 交叉脱氢偶联是有机合成中常用 的构建碳一硫和硫-杂原子键的方法 ${ }^{[7]}$. Lei ${ }^{[8]}$ 和 $\mathrm{Guo}^{[9]}{ }^{\left[\mathrm{N}_{\mathrm{J}}\right.}$ 分别通过无催化剂和无氧化剂的电化学和无金属的需 氧光催化策略，发展了富电子芳烃与芳基硫酚的脱氢 $\mathrm{C}-\mathrm{H} / \mathrm{S}-\mathrm{H}$ 交叉偶联。

2015 年, Prabhu 课题组 ${ }^{[10]}$ 研究了过硫酸钾氧化的苯 并噻唑-2-硫酮和 1,3-二羰基化合物的交叉脱氢偶联实

\footnotetext{
* Corresponding author. E-mail: hwhuang@xtu.edu.cn

Received November 25, 2021; revised December 11, 2021; published online December 15, 2021.

Project supported by the National Natural Science Foundation of China (No. 22071211) and the Natural Science Foundation of Hunan Province (No. 2020JJ3032).

国家自然科学基金(No. 22071211)及湖南省自然科学基金(No. 2020JJ3032)资助项目.
} 
现 C-S 键构建的反应(Scheme 1, a), 该反应条件苛刻, 并且反应产率很低, 只有 $17 \%$. Prabhu 课题组 ${ }^{[11]}$ 研究了 碘催化的吡唑酮类与杂芳环硫酚(或二硫化物)反应来实 现 $\mathrm{C}-\mathrm{S}$ 键的构建 (Scheme 1, b), 该方法使用二甲基亚砜 作为氧化剂, 但是苯硫酚类物质的反应活性较低, 产率 并没有得到很大的改善. 关于氧化 $\mathrm{C}\left(\mathrm{sp}^{3}\right)-\mathrm{S}$ 键的形成, 同年, Lei 等 ${ }^{[12]}$ 报道了碘催化 1,3-二酮和硫酚氧化偶联形 成 $\beta$-二羰基硫醚的反应(Scheme 1, c), 该反应以过量的 过氧化二叔丁基(DTBP)作为氧化剂. 不久后, 该课题 组 ${ }^{[13]}$ 提出了 ${ }^{t} \mathrm{BuOH}$ 氧化 $\beta-\mathrm{C}\left(\mathrm{sp}^{3}\right)-\mathrm{H}$ 硫基化的反应 (Scheme 1, d). 在这两种体系中, DTBP 被用作氧化剂, 大大提高了硫醚化合物的产率. 2016 年, Wang 及其同 事 ${ }^{[14]}$ 描述了一种通过可见光诱导的二芳基二硫醚与浐 啶红直接 $\alpha-\mathrm{C}\left(\mathrm{sp}^{3}\right)-\mathrm{H}$ 硫代化反应来获得 $\alpha$-芳基硫醚的 方法(Scheme 1, e), 为 $\mathrm{C}-\mathrm{S}$ 键的构建提供了新的思路. 基于以上报道, 本课题组以 1,3-二羰基化合物和颈基化 合物为底物, 空气作为氧化剂, 在可见光催化条件下, 直接交叉脱氢偶联实现 $\mathrm{C}-\mathrm{S}$ 键的构建, 该体系操作简 单, 反应条件温和, 原子经济性高, 底物适用范围广泛 且环境友好, 为 $\mathrm{C}-\mathrm{S}$ 键的合成提供了更简便的方法.

(a)<smiles>[X][X]c1cccc2[nH]c(=S)[X]c12</smiles>

(b)<smiles>CC1=NN(c2ccccc2)C(=O)C1</smiles><smiles>[R]C1=CCCCC1SSC1C=CCC=C1</smiles><smiles></smiles>

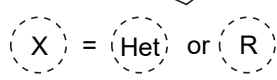

(c)

(d)<smiles>[R]C(=O)C([R])C([R])=O</smiles>

)<smiles>CC(C)(O)C[18F]</smiles>

(e)

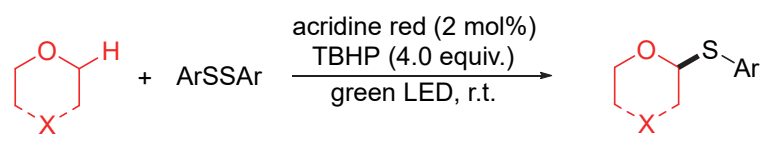

图式 $1 \mathrm{C}-\mathrm{S}$ 键的构建方法

Scheme 1 Construction method of $\mathrm{C}-\mathrm{S}$ bond

\section{1 结果与讨论}

首先，以乙酰丙酮(1a, 3 equiv.)和 4-氯苯硫酚 $(\mathbf{2} \mathbf{a}, 3$ $\mathrm{mmol}$ )作为模板底物, 探索最佳的反应条件. 如表 1 所 示, 刚开始反应在 $7 \mathrm{~W}$ 蓝光照射环境下进行, 以玫瑰红 为光催化剂, 乙腈作为溶剂, 探究添加剂对反应的影响. 当四丁基溴化铵(TBAB)作为添加剂时, 预期产物的产 率只有 15\%(表 1, Entry 1), 而含碘添加剂对产率有所提 高, 四丁基碘化铵(TBAI)使产率提高到 50\%(表 1, Entry $1)$, 碘单质参与的反应也以 $87 \%$ 的产率得到目标产物 (表 1, Entry 5), 而碘化钠的效果最佳(表 1, Entries 2 6), 目标产物 3a 的分离收率为 $93 \%$. 值得注意的是，改变底 物投料比为 2: 1 时(表 1, Entry 7), 产率将下降到 60\%. 接着对光催化剂进行笁选, 结果表明, 玫瑰红的催化效 果最好(表 1, Entries $8 \sim 10$ ), $\mathrm{Ru}(\text { bpy })_{3} \mathrm{Cl}_{2} \bullet 6 \mathrm{H}_{2} \mathrm{O} 、 \operatorname{Ir}($ ppy) 3 、 Eosin Y 均使产率有所降低. 根据这些结果, 篮选了一 系列溶剂, 将乙腈换成二氯甲烷或丙酮等溶剂时, 收率 均下降(表 1, Entries 11 13), 故乙腈作为溶剂时效果最 佳. 改变添加剂的用量, 当碘化钠的量改变为 0.5 和 1.5 equiv.时, 收率分别下降至 37\%和 71\%(表 1, Entries 14, 15). 稍后又进行了控制性试验, 实验结果表明, 光催化 剂和光照是该反应的必要条件, 否则不能检测到目标

表 1 条件优化表格

Table 1 Optimization on reaction conditions

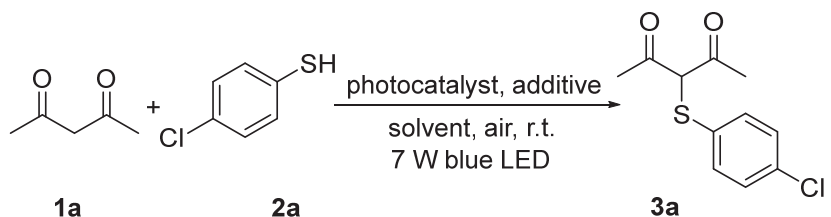

\begin{tabular}{clccc}
\hline Entry & \multicolumn{1}{c}{ Photocatalyst } & Additive & Solvent & Yield $^{a} \%$ \\
\hline 1 & Rose Bengal & TBAB & $\mathrm{CH}_{3} \mathrm{CN}$ & 15 \\
2 & Rose Bengal & TBAI & $\mathrm{CH}_{3} \mathrm{CN}$ & 50 \\
3 & Rose Bengal & $\mathrm{NH} \mathrm{N}_{4}$ & $\mathrm{CH}_{3} \mathrm{CN}$ & 38 \\
4 & Rose Bengal & $\mathrm{KI}$ & $\mathrm{CH}_{3} \mathrm{CN}$ & 43 \\
5 & Rose Bengal & $\mathrm{I} 2$ & $\mathrm{CH}_{3} \mathrm{CN}$ & 87 \\
6 & Rose Bengal & $\mathrm{NaI}$ & $\mathrm{CH}_{3} \mathrm{CN}$ & $93,78^{b}$ \\
$7^{c}$ & Rose Bengal & $\mathrm{NaI}$ & $\mathrm{CH}_{3} \mathrm{CN}$ & 60 \\
8 & Ru(bpy) ${ }_{3} \mathrm{Cl}_{2} \bullet 6 \mathrm{H}_{2} \mathrm{O}$ & $\mathrm{NaI}$ & $\mathrm{CH}_{3} \mathrm{CN}$ & 45 \\
9 & Ir(ppy) & $\mathrm{NaI}$ & $\mathrm{CH}_{3} \mathrm{CN}$ & 32 \\
10 & Eosin Y & $\mathrm{NaI}$ & $\mathrm{CH}_{3} \mathrm{CN}$ & 26 \\
11 & Rose Bengal & $\mathrm{NaI}$ & $\mathrm{CH}_{2} \mathrm{Cl}$ & 80 \\
12 & Rose Bengal & $\mathrm{NaI}$ & $\mathrm{Acetone}^{2}$ & 81 \\
13 & Rose Bengal & $\mathrm{NaI}$ & $\mathrm{DCE}^{2}$ & 78 \\
$14^{d}$ & Rose Bengal & $\mathrm{NaI}$ & $\mathrm{CH}_{3} \mathrm{CN}$ & 37 \\
$15^{e}$ & Rose Bengal & $\mathrm{NaI}$ & $\mathrm{CH}_{3} \mathrm{CN}$ & 71 \\
16 & - & $\mathrm{NaI}$ & $\mathrm{CH}_{3} \mathrm{CN}$ & nd \\
17 & Rose Bengal & - & $\mathrm{CH}_{3} \mathrm{CN}$ & 5 \\
$18^{f}$ & Rose Bengal & $\mathrm{NaI}$ & $\mathrm{CH}_{3} \mathrm{CN}$ & nd \\
$19^{g}$ & Rose Bengal & $\mathrm{NaI}$ & $\mathrm{CH}_{3} \mathrm{CN}$ & 10 \\
\hline
\end{tabular}

${ }^{a}$ Reaction conditions: 1a (3 equiv.), 2a $(0.3 \mathrm{mmol})$, photocatalyst $(1 \mathrm{~mol} \%)$, additive ( 1 equiv.), solvent ( $2 \mathrm{~mL}$ ), air, $7 \mathrm{~W}$ blue LED, r.t., $48 \mathrm{~h}$, isolated yield was given. ${ }^{b}$ With $1.5 \mathrm{~mL}$ of solvent. ${ }^{c} 2$ equiv. of $1 \mathrm{a}$ was used. ${ }^{d}$ With 0.5 equiv. of NaI. ${ }^{e}$ With 1.5 equiv. of NaI. ${ }^{f}$ In dark. ${ }^{g}$ Under $\mathrm{N}_{2}$ atmosphere. 
产物, 而添加剂和氧化环境则能大大提高原子经济效益

(表 1, Entries 16〜19).

在优化的反应条件下, 我们系统地研究了底物的普 适性. 首先, 探究了与乙酰丙酮发生偶联反应的颈基化 合物的适用范围(Scheme 2). 令人惊喜的是, 单取代或 多取代苯硫酚均能与乙酰丙酮发生反应, 卤原子和烷基 等取代的底物均以良好的收率得到目标产物 $\mathbf{3 a} \sim \mathbf{3 m}$; 该反应中吸电子基团或供电子基团的取代对偶联反应 没有明显影响, 空间效应微弱, 所以 2-萗硫酚也表现出 良好的耐受性, 收率为 $58 \%$ 的 $3 \mathrm{n}$. 茮硫醇、环己硫醇及 1-丙硫醇等脂肪族硫醇均能与乙酰丙酮 $(\mathbf{2 0} \sim \mathbf{2 q})$ 反应, 生成目标产物 3o 3q.

接下来, 在优化条件下考察了 4-氯苯硫酚与各类羰 基化合物的氧化脱氢偶联反应. 乙基和芳基取代的 1,3二羰基化合物以良好的产率获得目标产物 $3 \mathrm{r}$ 和 $3 \mathrm{~s}$. 同 样 5,5-二甲基-1,3-环已二酮也表现出了良好的选择性. 脂类化合物同样被证实适合该反应，乙酰乙酸乙酯和甲 基乙二酸二乙酯与硫酚偶联分别以 $59 \%$ 和 $42 \%$ 的产率 得到 $3 \mathbf{u}$ 和 $3 \mathbf{v}$. 此外丙二酸二甲酯得到了双偶联产物 $3 \mathbf{w}$, 产率为 $68 \%$.

在成功构建各种 $\mathrm{C}-\mathrm{S}$ 键的鼓励下, 随后进行了合
成 $3 \mathbf{a}$ 的放大量反应. 实验发现, 将硫酚的量放大至 10 $\mathrm{mmol}$ ，也体现了良好的反应性，3a 的产率高达 $65 \%$ (Scheme 3, a). 此外, 硫醚化产物 $\mathbf{3 a}$ 与亲电试剂茮基溴 和苯肼等的反应均能以良好的产率获得相应的季碳化 合物及杂化吡啶衍生物，证明了该反应在应用方面也有 广泛的适用性 (Scheme $3, \mathrm{~b} \sim \mathrm{d}$ ). 遗憾的是, 实验结果显 示放基杂芳烃不适用于该体系.

接下来，为了探索反应机理，设计了几个控制实验 并进行了验证(Scheme 4). 首先, 在合成 3a 的模型反应 中加入 2 equiv. 的自由基捕获剂 2,2,6,6-四甲基哌啶氧基 (TEMPO), 只能检测到极少量的目标产物, 且并无其他 副产物; 当加入 2 equiv. 的 1,1-二苯乙烯(1,1-diphenylethylene)到反应体系中时，除了得到 $41 \%$ 的预期产物， 还检测到了 $39 \%$ 的硫烷 7; 这两个实验说明反应过程中 可能产生了硫自由基. 当 2 equiv. 的三乙烯乙二胺 $(\mathrm{DABCO})$ 加入反应只能检测到微量的 $\mathbf{3 a}$, 则说明反应 中可能有单线态氧中间体存在. 此外, 在标准条件下, 乙酰丙酮与二芳基二硫醚 $\mathbf{8}$ 反应时，获得 $80 \%$ 收率的相 应产物 3a，表明 8 可能是反应的中间体. 对照实验的结 果证实了光照和碘添加剂的必要性.
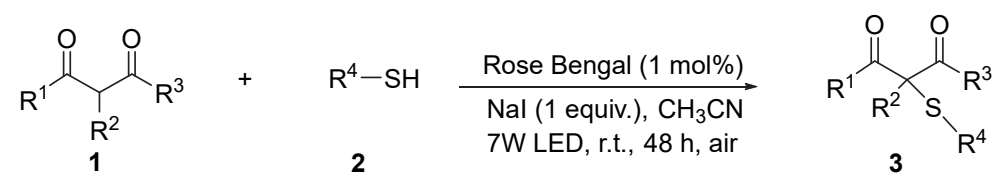

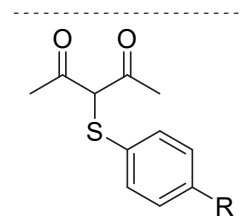

3a, $\mathrm{R}=\mathrm{Cl}, 93 \%$

3b, $R=F, 75 \%$

3c, $\mathrm{R}=\mathrm{Br}, 70 \%$

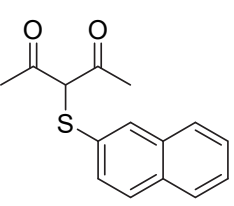

3 n, $58 \%$

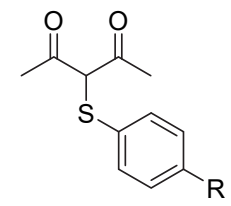

3d, $\mathrm{R}=\mathrm{CH}_{3}, 72 \%$

3e, $\mathrm{R}={ }^{t} \mathrm{Bu}, 70 \%$

3f, $\mathrm{R}=\mathrm{OCH}_{3}, 89 \%$

$3 g, \mathrm{R}=\mathrm{NO}_{2}, 65 \%$

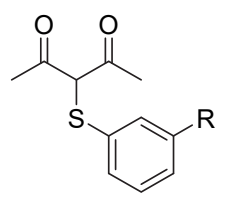

3h, $\mathrm{R}=\mathrm{CH}_{3}, 71 \%$

3i, $\mathrm{R}=\mathrm{F}, 56 \%$

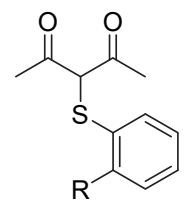

3j, $\mathrm{R}=\mathrm{CH}_{3}, 67 \%$

3k, R = F, 77\%

3I, $\mathrm{R}=\mathrm{Br}, 84 \%$

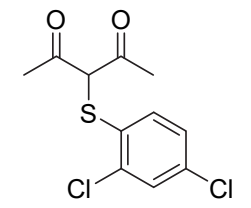

$3 \mathrm{~m}, 97 \%$

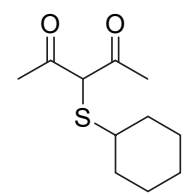

3p, $70 \%$<smiles>CCCSC(C(C)=O)C(C)=O</smiles>

$3 q, 25 \%$<smiles>CCC(=O)C(Sc1ccc(Cl)cc1)C(=O)CC</smiles>

$3 r, 86 \%$<smiles>COc1ccc(C(=O)C(Sc2ccc(Cl)cc2)C(=O)c2ccc(C(C)(C)C)cc2)cc1</smiles>

3s, $74 \%$<smiles>CC1(C)CC(=O)C(Sc2ccc(Cl)cc2)C(=O)C1</smiles>

3t, $90 \%$<smiles>CCOC(=O)C(Sc1ccc(Cl)cc1)C(C)=O</smiles>

$3 u, 59 \%$<smiles>CCOC(=O)C(C)(Sc1ccc(Cl)cc1)C(=O)OCC</smiles>

$3 v, 42 \%$

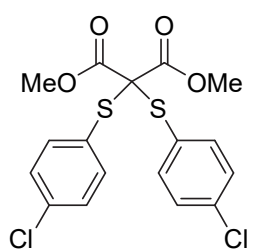

$3 w, 68 \%$

图式 2 羰基和硫酚的底物范围

Scheme 2 Substrate scope of carbonyls and thiophenols 
(a)<smiles>CC(=O)CC(C)=O</smiles>

2a $(10 \mathrm{mmol})$

1a (3 equiv.)<smiles>Sc1ccc(Cl)cc1</smiles>

Rose Bengal (1 mol\%), Nal (1 equiv.) $\mathrm{CH}_{3} \mathrm{CN}(50 \mathrm{~mL})$ air, r.t., $7 \mathrm{~W}$ blue LED

(b)

$3 a+$<smiles>[B]Cc1ccccc1</smiles>

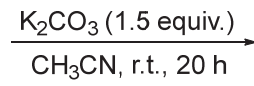<smiles>CC(=O)C(Cc1ccccc1)(Sc1ccc(Cl)cc1)C(C)=O</smiles>

4, $53 \%$

(c)<smiles>NNc1ccccc1</smiles><smiles>Cc1nn(-c2ccccc2)c(C)c1Sc1ccc(Cl)cc1</smiles>

5, $80 \%$

(d) $3 \mathrm{~m}+\mathrm{HO}-\mathrm{NH}_{3} \mathrm{Cl} \frac{\mathrm{NaOAc}(3 \text { equiv. })}{\mathrm{MeOH}(2 \mathrm{~mL}), 10 \mathrm{~h}}$<smiles>Cc1noc(C)c1Sc1ccc(Cl)cc1Cl</smiles>

$6,60 \%$

图式 3 克级规模反应和合成应用

Scheme 3 Gram scale reaction and applications<smiles>CC(=O)CC(C)=O</smiles><smiles>CC(=O)CC(C)=O</smiles>

$1 \mathrm{a}$

$2 a$

$41 \%$

$7,39 \%$<smiles>CC(=O)C(Sc1ccc(Cl)cc1)C(C)=O</smiles>

3a, $65 \%$<smiles>CC(=O)CC(C)=O</smiles>

$1 a$ $2 a$<smiles>CC(=O)C(Sc1ccc(Cl)cc1)C(C)=O</smiles><smiles>CC(=O)CC(C)=O</smiles>

图式 4 控制性实验

Scheme 4 Control experiments

基于上述研究和相关报道, 我们提出了一种可能的 机理(Scheme 5). 首先, 玫瑰红(RB)被蓝光照射形成激 发态 $\left(\mathrm{RB}^{*}\right), \mathrm{O}_{2}$ 通过该光催化剂激发态的能量转移形成 了单线态氧 $\left({ }^{1} \mathrm{O}_{2}\right)$, 与此同时, 激发态的光催化剂返回基 态. 接着, 单线态氧 $\left({ }^{1} \mathrm{O}_{2}\right)$ 氧化 4-氯苯基硫酚(2a)使其脱
去质子生成含硫自由基 9. 随后, 乙酰丙酮(1a)加成到含 硫自由基 9 上得到中间体 11, 该中间体 11 继而被氧化 形成碳正离子 12 , 碳正离子脱质子化后形成了目标产 物 3a. 


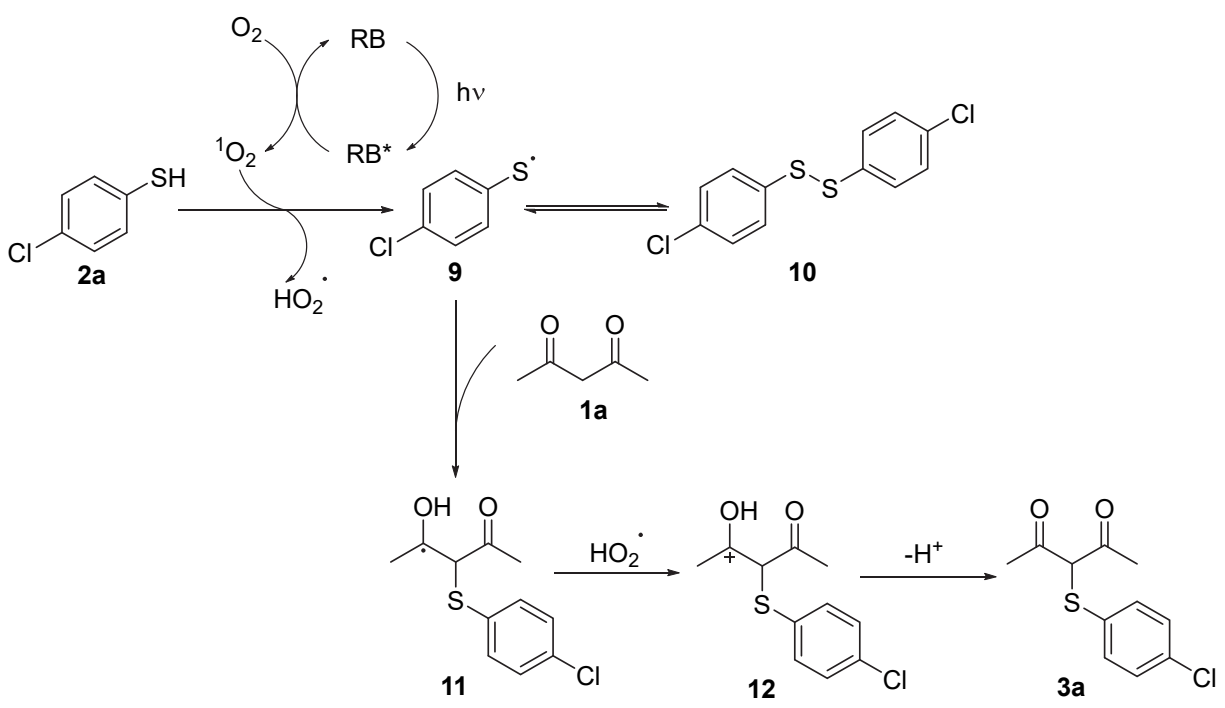

图式 5 可能的机理

Scheme 5 Possible mechanism

\section{2 结论}

以玫瑰红作为光催化剂, 碘化钠为添加剂, 空气为 氧化剂, 探索了羰基化合物与硫基化合物在光照条件下 氧化脱氢偶联构建 $\mathrm{C}-\mathrm{S}$ 键的反应, 考察了一系列不同 的羰基和硫源底物, 高选择性地得到兼容性多样的硫醚 产物, 并探索了其工业化的实用性, 为 $\mathrm{C}-\mathrm{S}$ 键的直接 构建提供了一种新的光/非金属协同催化体系. 毫无疑 问，可见光催化将会在更多的领域有着更多的应用.

\section{3 实验部分}

\section{1 仪器与试剂}

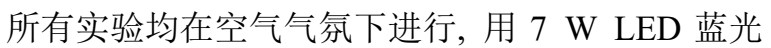
灯作为光源. 除特别说明, 所有原料均来自商业购买, 并直接使用, 使用薄层层析(TLC) 跟踪反应进行程度. 柱层析使用 200 300 目硅胶, TLC 使用 GF254 硅胶板. ${ }^{1} \mathrm{H}$ NMR 和 ${ }^{13} \mathrm{C}$ NMR 采用 Bruker-AV 核磁共振仪测试, 频率分别为 $400 \mathrm{MHz}$ 和 $100 \mathrm{MHz}$, 以 $\mathrm{CDCl}_{3}$ 为溶剂, TMS 为内标. 高分辨质谱采用 Thermo Scientific LTQ Orbitrap XL (ESI)质谱仪测定.

\section{2 实验方法}

以模板反应为例, 反应在烘箱干燥的玻璃器血中进 行. 称取 $0.3 \mathrm{mmol}$ 对氯苯硫酚、 $0.9 \mathrm{mmol}$ 乙酰丙酮、 0.3 $\mathrm{mmol}$ 碘化钠和 $1 \mathrm{~mol} \%$ 的孟加拉玫瑰红, 将其溶解于 2 $\mathrm{mL}$ 的乙腈中, 在室温下, 用 $7 \mathrm{~W}$ 的 LED 灯照射, 再充 分摚拌 $48 \mathrm{~h}$. 反应结束后, 在反应液中加入水, 用乙酸 乙酯萃取三遍, 合并有机相并用无水硫酸镁干燥, 过滤, 再减压蒸馏, 通过薄层层析(石油醚/乙酸乙酯, $V: V=$
$50 ： 1$ )得到目标产物, 产率为 $93 \%$.

3-((4-氯苯基)硫代)-4-着基戊-3-烯-2-酮(3a): 白色 固体, 产率 90\%. m.p. 66 $67{ }^{\circ} \mathrm{C}$ (lit. ${ }^{[15]} 69 \sim 71{ }^{\circ} \mathrm{C}$ ); ${ }^{1} \mathrm{H}$ NMR (400 MHz, $\left.\mathrm{CDCl}_{3}\right) \delta: 17.28(\mathrm{~s}, 1 \mathrm{H}), 7.25$ (d, $J=8.8$ $\mathrm{Hz}, 2 \mathrm{H}), 7.01$ (d, $J=8.4 \mathrm{~Hz}, 2 \mathrm{H}), 2.32$ (s, 6H); ${ }^{13} \mathrm{C}$ NMR $\left(100 \mathrm{MHz}, \mathrm{CDCl}_{3}\right) \delta: 198.3,136.4,131.1,129.3,125.9$, $101.4,24.4$.

3-((4-氟苯基)硫代)-4-着基戊-3-烯-2-酮(3b $)^{[15]}$ : 无 色油状物, 产率 75\%. ${ }^{1} \mathrm{H}$ NMR $\left(400 \mathrm{MHz}, \mathrm{CDCl}_{3}\right) \delta$ : 17.25 (s, 1H), 7.06 (dd, $J=9.2,5.2 \mathrm{~Hz}, 2 \mathrm{H}), 6.99$ (t, $J=$ $8.6 \mathrm{~Hz}, 2 \mathrm{H}), 2.34(\mathrm{~s}, 6 \mathrm{H}) ;{ }^{13} \mathrm{C} \mathrm{NMR}\left(100 \mathrm{MHz}, \mathrm{CDCl}_{3}\right) \delta$ : $198.2,162.3,159.7,132.8,126.6,126.5,116.5,116.2$, 102.2, 24.4 .

3-((4-溴苯基)硫代)-4-羟基戊-3-烯-2-酮(3c)：白色 固体，产率 70\%. m.p. 69 70 ${ }^{\circ} \mathrm{C}$ (lit. ${ }^{[15]} 70 \sim 71{ }^{\circ} \mathrm{C}$ ); ${ }^{1} \mathrm{H}$ NMR (400 MHz, $\left.\mathrm{CDCl}_{3}\right) \delta: 17.29$ (s, 1H), 7.39(d, $J=8.4$ $\mathrm{Hz}, 2 \mathrm{H}), 6.95$ (d, $J=8.4 \mathrm{~Hz}, 2 \mathrm{H}), 2.32$ (s, 6H); ${ }^{13} \mathrm{C} \mathrm{NMR}$ $\left(100 \mathrm{MHz}, \mathrm{CDCl}_{3}\right) \delta: 198.4,137.1,132.2,126.2,118.8$, 101.2, 24.4 .

4-差基-3-(对甲苯基硫代)戊-3-烯-2-酮(3d)：白色固 体, 产率 72\%. m.p. 39 41 ${ }^{\circ} \mathrm{C}$ (lit. ${ }^{[15]}$ 无色油状物); ${ }^{1} \mathrm{H}$ NMR (400 MHz, $\left.\mathrm{CDCl}_{3}\right) \delta$ : $17.25(\mathrm{~s}, 1 \mathrm{H}), 7.10$ (d, $J=8.4$ $\mathrm{Hz}, 2 \mathrm{H}), 6.99$ (d, $J=8 \mathrm{~Hz}, 2 \mathrm{H}), 2.34$ (s, 6H), 2.31(s, 3H); ${ }^{13} \mathrm{C}$ NMR $\left(100 \mathrm{MHz}, \mathrm{CDCl}_{3}\right) \delta: 198.2,135.1,134.2,130.0$, 124.9, 102.1, 24.4, 20.9.

3-((4-(叔丁基)苯基)硫代)-4-羟基戊-3-烯-2-酮(3e): 白色固体，产率 70\%. m.p. 99 100 ${ }^{\circ} \mathrm{C}$ (lit. ${ }^{[15]}$ 104 $\left.106{ }^{\circ} \mathrm{C}\right) ;{ }^{1} \mathrm{H}$ NMR (400 MHz,) $\delta: 17.27$ (s, 1H), 7.30 (d, $J=8.4 \mathrm{~Hz}, 2 \mathrm{H}), 7.02(\mathrm{~d}, J=8.8 \mathrm{~Hz}, 2 \mathrm{H}), 2.35$ (s, 6H), 1.30 
$(\mathrm{s}, 9 \mathrm{H}) ;{ }^{13} \mathrm{C}$ NMR $\left(100 \mathrm{MHz}, \mathrm{CDCl}_{3}\right) \delta: 198.3,148.4$, 134.2, 126.3, 124.6, 102.0, 34.4, 31.3, 24.5.

4-差基-3-((4-甲氧基苯基)硫代)戊-3-烯-2-酮(3f): 白 色固体, 产率 $89 \%$. m.p. 84 86 ${ }^{\circ} \mathrm{C}$ (lit. ${ }^{[11]} 84 \sim 85{ }^{\circ} \mathrm{C}$ ); ${ }^{1} \mathrm{H}$ NMR (400 MHz, $\left.\mathrm{CDCl}_{3}\right) \delta: 17.20(\mathrm{~s}, 1 \mathrm{H}), 7.04(\mathrm{~d}, J=$ $8.8 \mathrm{~Hz}, 2 \mathrm{H}), 6.84$ (d, $J=8.8 \mathrm{~Hz}, 2 \mathrm{H}), 3.77$ (s, 3H), 3.36 (s, $6 \mathrm{H}) ;{ }^{13} \mathrm{C}$ NMR (100 MHz, $\left.\mathrm{CDCl}_{3}\right) \delta: 198.1,158.0,128.4$, 126.9, 115.0, 103.1, 55.4, 24.5.

4-差基-3-((4-硝基苯基)硫代)戊-3-烯-2-酮(3g)：黄 色固体, 产率 65\%. m.p. $110 \sim 112{ }^{\circ} \mathrm{C}$ (lit. ${ }^{[11]} 105 \sim$ $\left.108{ }^{\circ} \mathrm{C}\right) ;{ }^{1} \mathrm{H} \mathrm{NMR}\left(400 \mathrm{MHz}, \mathrm{CDCl}_{3}\right) \delta: 17.39$ (s, 1H), 8.14 (d, $J=8.4 \mathrm{~Hz}, 2 \mathrm{H}), 7.20$ (d, $J=8.8 \mathrm{~Hz}, 2 \mathrm{H}), 2.31$ (s, $6 \mathrm{H}) ;{ }^{13} \mathrm{C}$ NMR $\left(100 \mathrm{MHz}, \mathrm{CDCl}_{3}\right) \delta: 198.5,147.6,145.5$, 124.5, 124.3, 99.8, 24.3.

4-着基-3-(间甲基硫代)戊-3-烯-2-酮(3h): 无色油状 物, 产率 71\%. ${ }^{1} \mathrm{H}$ NMR (400 $\left.\mathrm{MHz}, \mathrm{CDCl}_{3}\right) \delta: 17.28(\mathrm{~s}$, $1 \mathrm{H}), 7.17(\mathrm{t}, J=7.6 \mathrm{~Hz}, 1 \mathrm{H}), 6.95(\mathrm{~d}, J=7.5 \mathrm{~Hz}, 1 \mathrm{H})$, $6.93 \sim 6.86(\mathrm{~m}, 2 \mathrm{H}), 2.34(\mathrm{~s}, 6 \mathrm{H}), 2.32(\mathrm{~s}, 3 \mathrm{H}) ;{ }^{13} \mathrm{C} \mathrm{NMR}$ $\left(100 \mathrm{MHz}, \mathrm{CDCl}_{3}\right) \delta: 198.3,139.1,137.6,129.1,126.2$, 125.2, 121.7, 101.7, 24.5, 21.5; HRMS (ESI) calcd for $\mathrm{C}_{12} \mathrm{H}_{15} \mathrm{O}_{2} \mathrm{~S}(\mathrm{M}+\mathrm{H})^{+}$223.0787, found 223.0795.

3-((3-氟苯基)硫代)-4-差基戊-3-烯-2-酮(3i) ${ }^{[15]}$ : 无色 油状物, 产率 $56 \%$. ${ }^{1} \mathrm{H} \mathrm{NMR}\left(400 \mathrm{MHz}, \mathrm{CDCl}_{3}\right) \delta: 17.32$ $(\mathrm{s}, 1 \mathrm{H}), 7.27 \sim 7.21(\mathrm{~m}, 1 \mathrm{H}), 6.87(\mathrm{dd}, J=8.0,0.8 \mathrm{~Hz}, 1 \mathrm{H})$, $6.85 \sim 6.76(\mathrm{~m}, 2 \mathrm{H}), 2.33(\mathrm{~s}, 6 \mathrm{H}) ;{ }^{13} \mathrm{C} \mathrm{NMR}(100 \mathrm{MHz}$, $\left.\mathrm{CDCl}_{3}\right) \delta: 198.4,164.6,162.2,140.5,130.4,130.6,130.5$, $120.2,120.2,112.4,112.1,111.4,101.0,24.4$.

4-羟基-3-(邻甲苯基硫代)戊-3-烯-2-酮(3j): 白色固 体, 产率 67\%. m.p. 66 68 ${ }^{\circ} \mathrm{C}$ (lit. ${ }^{[15]} 67 \sim 69{ }^{\circ} \mathrm{C}$ ); ${ }^{1} \mathrm{H}$ NMR (400 MHz, $\left.\mathrm{CDCl}_{3}\right) \delta: 17.33(\mathrm{~s}, 1 \mathrm{H}), 7.14$ (dd, $J=$ 7.2, $12.4 \mathrm{~Hz}, 2 \mathrm{H}), 7.06$ (dd, $J=7.3,6.9 \mathrm{~Hz}, 1 \mathrm{H}), 6.84$ (d, $J=8.0 \mathrm{~Hz}, 1 \mathrm{H}), 2.38(\mathrm{~s}, 3 \mathrm{H}), 2.31(\mathrm{~s}, 6 \mathrm{H}) ;{ }^{13} \mathrm{C} \mathrm{NMR}(100$ $\left.\mathrm{MHz}, \mathrm{CDCl}_{3}\right) \delta: 198.4,136.6,134.4,130.4,126.8,124.8$, $122.7,100.8,24.3,19.6$.

3-((2-氟苯基)硫代)-4-羟基戊-3-烯-2-酮(3k): 白色 固体, 产率 77\%. m.p. 55 56 ${ }^{\circ} \mathrm{C}$ (lit. ${ }^{[15]} 54 \sim 56{ }^{\circ} \mathrm{C}$ ); ${ }^{1} \mathrm{H}$ NMR (400 MHz, $\left.\mathrm{CDCl}_{3}\right) \delta: 17.34(\mathrm{~s}, 1 \mathrm{H}), 7.15 \sim 7.11(\mathrm{~m}$, $1 \mathrm{H}), 7.09 \sim 7.04(\mathrm{~m}, 2 \mathrm{H}), 6.96 \sim 6.93(\mathrm{~m}, 1 \mathrm{H}), 2.34(\mathrm{~s}$, $6 \mathrm{H}) ;{ }^{13} \mathrm{C}$ NMR $\left(100 \mathrm{MHz}, \mathrm{CDCl}_{3}\right) \delta: 198.5,160.5,158.1$, $126.9,126.8,126.1,126.1,125.0,124.9,124.8,124.7$, $115.8,115.6,99.7,24.4$.

3-((2-溴苯基)硫代)-4-羟基戊-3-烯-2-酮(31): 白色固 体, 产率 84\%. m.p. 82 84 ${ }^{\circ} \mathrm{C}$ (lit. ${ }^{[15]} 84 \sim 85{ }^{\circ} \mathrm{C}$ ); ${ }^{1} \mathrm{H}$ NMR (400 MHz, $\left.\mathrm{CDCl}_{3}\right) \delta: 17.38(\mathrm{~s}, 1 \mathrm{H}), 7.52$ (dd, $J=$ $7.9,1.0 \mathrm{~Hz}, 1 \mathrm{H}), 7.26 \sim 7.17(\mathrm{~m}, 1 \mathrm{H}), 7.00(\mathrm{td}, J=7.7,1.4$
$\mathrm{Hz}, 1 \mathrm{H}), 6.83(\mathrm{dd}, J=8.0,1.4 \mathrm{~Hz}, 1 \mathrm{H}), 2.30(\mathrm{~s}, 6 \mathrm{H}) ;{ }^{13} \mathrm{C}$ NMR $\left(100 \mathrm{MHz}, \mathrm{CDCl}_{3}\right) \delta: 198.6,138.6,133.2,128.0$, 126.3, 124.4, 120.1, 101.0, 23.3.

3-((2,4-二氯苯基)硫代)-4-羟基戊-3-烯-2-酮(3m): 白色固体，产率 97\%. m.p. 45 47 ${ }^{\circ} \mathrm{C}$ (lit. ${ }^{[15]}$ 无色油状 物); ${ }^{1} \mathrm{H}$ NMR (400 MHz, $\left.\mathrm{CDCl}_{3}\right) \delta: 17.39$ (s, 1H), 7.37 (d, $J=2.0 \mathrm{~Hz}, 1 \mathrm{H}), 7.17(\mathrm{dd}, J=8.4,2.0 \mathrm{~Hz}, 1 \mathrm{H}), 6.78(\mathrm{~d}, J=$ $8.8 \mathrm{~Hz}, 1 \mathrm{H}), 2.30(\mathrm{~s}, 6 \mathrm{H}) ;{ }^{13} \mathrm{C} \mathrm{NMR}\left(100 \mathrm{MHz}, \mathrm{CDCl}_{3}\right) \delta$ : 198.6, 135.5, 131.2, 131.1, 129.7, 127.7, 125.1, 99.8, 24.3.

4-羟基-3-(菜-2-基硫代)戊-3-烯-2-酮(3n): 白色固 体, 产率 58\%. m.p. 86 88 ${ }^{\circ} \mathrm{C}$ (lit. ${ }^{[15]} 88 \sim 89{ }^{\circ} \mathrm{C}$ ); ${ }^{1} \mathrm{H}$ NMR (400 MHz, $\left.\mathrm{CDCl}_{3}\right) \delta: 17.38(\mathrm{~s}, 1 \mathrm{H}), 7.78(\mathrm{t}, J=8.0$ $\mathrm{Hz}, 2 \mathrm{H}), 7.72(\mathrm{~d}, J=8.0 \mathrm{~Hz}, 1 \mathrm{H}), 7.50 \sim 7.40(\mathrm{~m}, 3 \mathrm{H})$, $7.28 \sim 7.25(\mathrm{~m}, 1 \mathrm{H}), 2.38(\mathrm{~s}, 6 \mathrm{H}) ;{ }^{13} \mathrm{C} \mathrm{NMR}(100 \mathrm{MHz}$, $\left.\mathrm{CDCl}_{3}\right) \delta: 198.5,135.3,134.0,131.5,128.9,127.8,126.9$, $126.8,125.4,123.7,121.8,101.5,24.5$.

3-(苠基硫)-4-羟基戊-3-烯-2-酮(30) ${ }^{[16]}$ : 无色油状物, 产率 67\%. ${ }^{1} \mathrm{H}$ NMR $\left(400 \mathrm{MHz}, \mathrm{CDCl}_{3}\right) \delta: 17.22(\mathrm{~s}, 1 \mathrm{H})$, $7.30 \sim 7.22(\mathrm{~m}, 3 \mathrm{H}), 7.11(\mathrm{~d}, J=7.4 \mathrm{~Hz}, 2 \mathrm{H}), 3.62(\mathrm{~s}, 2 \mathrm{H})$, 2.09 (s, 6H); ${ }^{13} \mathrm{C}$ NMR (100 MHz, $\left.\mathrm{CDCl}_{3}\right) \delta: 198.4,137.7$, 129.1, 128.6, 127.2, 103.1, 41.0, 24.1.

3-(环己基硫代)-4-羟基戊-3-烯-2-酮(3p): 无色油状 物, 产率 70\%. ${ }^{1} \mathrm{H}$ NMR (400 $\left.\mathrm{MHz}, \mathrm{CDCl}_{3}\right) \delta: 17.18(\mathrm{~s}$, $1 \mathrm{H}), 2.57 \sim 2.52(\mathrm{~m}, 1 \mathrm{H}), 2.41(\mathrm{~s}, 6 \mathrm{H}), 1.90 \sim 1.63(\mathrm{~m}$, $6 \mathrm{H}), 1.30 \sim 1.21(\mathrm{~m}, 4 \mathrm{H}) ;{ }^{13} \mathrm{C}$ NMR $\left(100 \mathrm{MHz}, \mathrm{CDCl}_{3}\right) \delta$ : 197.8, 103.7, 48.4, 33.1, 26.1, 25.7, 24.8; HRMS (ESI) calcd for $\mathrm{C}_{11} \mathrm{H}_{19} \mathrm{O}_{2} \mathrm{~S}(\mathrm{M}+\mathrm{H})^{+}$215.1100, found 215.1101.

4-羟基-3-(丙硫基)戊-3-烯-2-酮(3q): 无色油状物, 产率 25\%. ${ }^{1} \mathrm{H} \mathrm{NMR}\left(400 \mathrm{MHz}, \mathrm{CDCl}_{3}\right) \delta: 17.10(\mathrm{~s}, 1 \mathrm{H})$, $2.47(\mathrm{t}, J=7.4 \mathrm{~Hz}, 2 \mathrm{H}), 2.43(\mathrm{~s}, 6 \mathrm{H}), 1.57$ (dd, $J=14.8$, $7.2 \mathrm{~Hz}, 2 \mathrm{H}), 0.99$ (t, $J=7.2 \mathrm{~Hz}, 3 \mathrm{H}) ;{ }^{13} \mathrm{C} \mathrm{NMR}(100 \mathrm{MHz}$, $\left.\mathrm{CDCl}_{3}\right) \delta: 197.5,104.7,38.9,24.6,22.5,13.5$; HRMS (ESI) calcd for $\mathrm{C}_{8} \mathrm{H}_{16} \mathrm{O}_{2} \mathrm{~S}(\mathrm{M}+\mathrm{H})^{+}$175.0787, found 175.0789 .

4-((4-氯苯基)硫代)-5-羟基庚-4-烯-3-酮(3r): 白色 固体, 产率 $86 \%$. m.p. $38 \sim 39{ }^{\circ} \mathrm{C} ;{ }^{1} \mathrm{H}$ NMR (400 MHz, $\left.\mathrm{CDCl}_{3}\right) \delta: 17.50(\mathrm{~s}, 1 \mathrm{H}), 7.23(\mathrm{~d}, J=8.8 \mathrm{~Hz}, 2 \mathrm{H}), 7.00(\mathrm{~d}$, $J=8.8 \mathrm{~Hz}, 2 \mathrm{H}), 2.70$ (s, 4H), 1.09 (t, $J=7.4 \mathrm{~Hz}, 6 \mathrm{H}) ;{ }^{13} \mathrm{C}$ NMR $\left(100 \mathrm{MHz}, \mathrm{CDCl}_{3}\right) \delta: 201.3,136.9,130.9,129.3$, 125.7, 99.8, 30.0, 9.6; HRMS (ESI) calcd for $\mathrm{C}_{13} \mathrm{H}_{16} \mathrm{ClO}_{2} \mathrm{~S}$ $(\mathrm{M}+\mathrm{H})^{+}$271.0554, found 271.0550 .

2-((4-氯苯基)硫代)-5,5-二甲基环己烷-1,3-二酮(3s): 白色固体，产率 90\%. m.p. 138 $139{ }^{\circ} \mathrm{C} ;{ }^{1} \mathrm{H}$ NMR (400 $\left.\mathrm{MHz} \mathrm{CDCl}_{3}\right) \delta: 8.12(\mathrm{~s}, 1 \mathrm{H}), 7.17(\mathrm{~d}, J=8.4 \mathrm{~Hz}, 2 \mathrm{H})$, 7.09 (d, $J=8.8 \mathrm{~Hz}, 2 \mathrm{H}), 2.48(\mathrm{~s}, 4 \mathrm{H}), 1.10(\mathrm{~s}, 6 \mathrm{H}) ;{ }^{13} \mathrm{C}$ 
NMR (100 MHz, $\left.\mathrm{CDCl}_{3}\right) \delta: 133.4,132.4,129.2,129.0$, 105.9, 54.1, 31.6, 28.3; HRMS (ESI) calcd for $\mathrm{C}_{14} \mathrm{H}_{16} \mathrm{Cl}-$ $\mathrm{O}_{2} \mathrm{~S}(\mathrm{M}+\mathrm{H})^{+}$283.0554, found 283.0556.

1-(4-(叔丁基)苯基)-2-(4-氯苯基)硫代)-3-(4-甲氧基 苯基)丙烷-1,3-二酮(3t): 白色固体，产率 74\%. m.p. $134 \sim 136{ }^{\circ} \mathrm{C}$; ${ }^{1} \mathrm{H}$ NMR $\left(400 \mathrm{MHz}, \mathrm{CDCl}_{3}\right) \delta: 7.96$ (d, $J=$ $8.4 \mathrm{~Hz}, 2 \mathrm{H}), 7.90$ (d, $J=8.0 \mathrm{~Hz}, 2 \mathrm{H}), 7.41$ (d, $J=8.0 \mathrm{~Hz}$, $2 \mathrm{H}), 7.36(\mathrm{~d}, J=8.0 \mathrm{~Hz}, 2 \mathrm{H}), 7.22$ (d, $J=8.0 \mathrm{~Hz}, 2 \mathrm{H}), 6.88$ $(\mathrm{d}, J=8.0 \mathrm{~Hz}, 2 \mathrm{H}), 5.95$ (s, 1H), $3.84(\mathrm{~s}, 3 \mathrm{H}), 1.30$ (s, 9H); ${ }^{13} \mathrm{C}$ NMR (100 MHz, $\left.\mathrm{CDCl}_{3}\right) \delta: 191.4,190.1,164.1,157.9$, $135.2,134.9,132.4,131.8,131.1,129.3,129.2$, 127.9, 125.8, 114.0, 65.1, 55.6, 35.2, 31.0; HRMS (ESI) calcd for $\mathrm{C}_{26} \mathrm{H}_{26} \mathrm{ClO}_{3} \mathrm{~S}(\mathrm{M}+\mathrm{H})^{+}$453.1286, found 453.1283.

3-((4-氯苯基)硫代)-4-着基-4-甲氧基-3-烯-2-酮 $(3 \mathbf{u})$ : 无色油状物, 产率 59\%. ${ }^{1} \mathrm{H}$ NMR (400 $\left.\mathrm{MHz}^{-} \mathrm{CDCl}_{3}\right) \delta$ : $13.86(\mathrm{~s}, 1 \mathrm{H}), 7.21$ (d, $J=8.4 \mathrm{~Hz}, 2 \mathrm{H}), 7.05$ (d, $J=8.4 \mathrm{~Hz}$, 2H), 4.21 (q, $J=7.1 \mathrm{~Hz}, 2 \mathrm{H}), 2.33$ (s, 3H), 1.91 (t, $J=7.2$ $\mathrm{Hz}, 3 \mathrm{H}) ;{ }^{13} \mathrm{C} \mathrm{NMR}\left(100 \mathrm{MHz}, \mathrm{CDCl}_{3}\right) \delta: 184.9,172.7$, 136.7, 130.9, 129.0, 126.8, 91.9, 61.8, 21.0, 14.1; HRMS (ESI) calcd for $\mathrm{C}_{12} \mathrm{H}_{14} \mathrm{ClO}_{3} \mathrm{~S}(\mathrm{M}+\mathrm{H})^{+}$273.0347, found 273.0348

2-((4-氯苯基)硫代)-2-甲基丙二酸二乙酯 $(3 v)$ : 白色 固体, 产率 42\%. m.p. 39 41 ${ }^{\circ} \mathrm{C} ;{ }^{1} \mathrm{H}$ NMR (400 MHz, $\left.\mathrm{CDCl}_{3}\right) \delta: 7.47(\mathrm{~d}, J=8.4 \mathrm{~Hz}, 2 \mathrm{H}), 7.29(\mathrm{~d}, J=8.4 \mathrm{~Hz}$, $2 \mathrm{H}), 4.23 \sim 4.15(\mathrm{~m}, 4 \mathrm{H}), 1.61(\mathrm{~s}, 3 \mathrm{H}), 1.23(\mathrm{t}, J=7.2 \mathrm{~Hz}$, $6 \mathrm{H}) ;{ }^{13} \mathrm{C}$ NMR $\left(100 \mathrm{MHz}, \mathrm{CDCl}_{3}\right) \delta: 169.1,138.4,136.4$, 129.0, 128.5, 62.3, 60.1, 22.2, 14.0; HRMS (ESI) calcd for $\mathrm{C}_{14} \mathrm{H}_{18} \mathrm{ClO}_{4} \mathrm{~S}(\mathrm{M}+\mathrm{H})^{+}$317.0609, found 317.0610.

2-((4-氯苯基)硫代)-2-甲基丙二酸二甲酯(3w): 白 色固体, 产率 $68 \%$. m.p. $127 \sim 129{ }^{\circ} \mathrm{C}$; ${ }^{1} \mathrm{H}$ NMR (400 $\left.\mathrm{MHz} \mathrm{CDCl}_{3}\right) \delta: 7.58(\mathrm{~d}, J=8.4 \mathrm{~Hz}, 4 \mathrm{H}), 7.35$ (d, $J=8.4$ $\mathrm{Hz}, 4 \mathrm{H}), 3.65$ (s, 6H); ${ }^{13} \mathrm{C}$ NMR (100 MHz, $\left.\mathrm{CDCl}_{3}\right) \delta$ : 165.6, 137.7, 137.0, 129.3, 127.9, 73.8, 53.7; HRMS (ESI) calcd for $\mathrm{C}_{17} \mathrm{H}_{15} \mathrm{Cl}_{2} \mathrm{O}_{4} \mathrm{~S}_{2}(\mathrm{M}+\mathrm{H})^{+}$416.9783, found 416.9786

3-芐基-3-((4-氯苯基)硫代)戊烷-2,4-二酮(4): 无色 油状物，产率 53\%。 ${ }^{1} \mathrm{H}$ NMR (400 $\left.\mathrm{MHz} \mathrm{CDCl}_{3},\right) \delta$ : $7.30 \sim 7.26(\mathrm{~m}, 9 \mathrm{H}), 3.24(\mathrm{~s}, 2 \mathrm{H}), 2.32(\mathrm{~s}, 6 \mathrm{H}) ;{ }^{13} \mathrm{C} \mathrm{NMR}$ $\left(100 \mathrm{MHz}, \mathrm{CDCl}_{3}\right) \delta: 201.2,137.2,136.5,135.1,129.8$, 129.6, 128.5, 127.6, 127.2, 77.5, 37.5, 27.9; HRMS (ESI) calcd for $\mathrm{C}_{18} \mathrm{H}_{18} \mathrm{ClO}_{2} \mathrm{~S}(\mathrm{M}+\mathrm{H})^{+}$333.0711, found 333.0702

4-((4-氯苯基)硫代)-3,5-二甲基-1-苯基- $1 H$-吡唑 $(5)^{[17]}$ : 无色油状物, 产率 $80 \%$. ${ }^{1} \mathrm{H}$ NMR (400 $\mathrm{MHz}$, $\left.\mathrm{CDCl}_{3}\right) \delta: 7.51 \sim 7.46(\mathrm{~m}, 4 \mathrm{H}), 7.40(\mathrm{~d}, J=6.4 \mathrm{~Hz}, 1 \mathrm{H})$,
7.19 (d, $J=8.0 \mathrm{~Hz}, 2 \mathrm{H}), 6.98$ (d, $J=8.0 \mathrm{~Hz}, 2 \mathrm{H}), 2.33$ (s, $3 \mathrm{H}), 2.28(\mathrm{~s}, 3 \mathrm{H}) ;{ }^{13} \mathrm{C}$ NMR $\left(100 \mathrm{MHz}, \mathrm{CDCl}_{3}\right) \delta: 153.1$, $144.2,139.6,136.9,130.7,129.3,129.0,128.0,126.6$, $124.7,105.7,12.1,11.6$.

4-((2,4-二氯苯基)硫代)-3,5-二甲基异噁唑(6): 白色 固体，产率 60\%. m.p. $67 \sim 69{ }^{\circ} \mathrm{C} ;{ }^{1} \mathrm{H}$ NMR $(400 \mathrm{MHz}$, $\left.\mathrm{CDCl}_{3}\right) \delta: 7.38(\mathrm{~d}, J=2.0 \mathrm{~Hz}, 1 \mathrm{H}), 7.09(\mathrm{dd}, J=8.6,2.2$ $\mathrm{Hz}, 1 \mathrm{H}), 6.55$ (d, $J=8.8 \mathrm{~Hz}, 1 \mathrm{H}), 2.45$ (s, 3H), 2.19 (s, $1 \mathrm{H}) ;{ }^{13} \mathrm{C}$ NMR $\left(100 \mathrm{MHz}, \mathrm{CDCl}_{3}\right) \delta: 174.5,162.5,134.1$, $131.7,131.5,129.7,127.6,126.4,102.5,11.6,10.1$; HRMS (ESI) calcd for $\mathrm{C}_{11} \mathrm{H}_{10} \mathrm{Cl}_{2} \mathrm{NOS}(\mathrm{M}+\mathrm{H})^{+} 273.9855$, found 273.9850

辅助材料(Supporting Information) 化合物 3 的 ${ }^{1} \mathrm{H}$ $\mathrm{NMR}$ 和 ${ }^{13} \mathrm{C} \mathrm{NMR}$ 谱图. 这些材料可以免费从本刊网站 (http://sioc-journal.cn/)上下载.

\section{References}

[1] (a) Dondoni, A. Angew. Chem., Int. Ed. 2008, 47, 8995. (b) Boyd, D. A. Angew. Chem., Int. Ed. 2016, 55, 15486.

(c) Pathak, A. K.; Pathak, V.; Seitz, L. E.; Suling, W. J.; Reynolds, R. C. J. Med. Chem. 2004, 47, 273.

(d) Chivers, T.; Elder, P. J. Chem. Soc. Rev. 2013, 42, 5996.

(e) Liu, H.; Jiang, X. Chem.-Asian. J. 2013, 8, 2546.

(f) Yu, Y.; Duan, W.; Lin, G.; Kang, G.; Wang, X.; Lei, F. Chin. J. Org. Chem. 2020, 40, 1647 (in Chinese).

(虞友培, 段文贵, 林桂汕, 康国强, 王晓宇, 雷福厚, 有机化学, 2020, 40, 1647.)

(g) He, M.; He, C.; Liu, L.; Ye, J.; Hu, A.; Chen, Y.; Xu, L.; Liu, A Chin. J. Org. Chem. 2020, 40, 2402 (in Chinese)

(何梅，贺超凡，刘玲，叶姣，胡艾希，陈云，许律捷，刘艾林， 有机化学, 2020, 40, 2402.)

(h) Yan, X.; Li, C.; Jin, Z.; Xu, X.; Chen, W.; Pan, Y. Chin. J. Org. Chem. 2020, 40, 3837 (in Chinese)

(问晓静, 李畅, 靳智雄, 徐孝菲, 陈维伟, 潘远江, 有机化学, 2020, 40, 3837.)

[2] (a) Chivers, T.; Elder, P. J. W. Chem. Soc. Rev. 2013, 42, 5996 (b) Liu, B.; Lim, C. H.; Miyake, G. M. J. Am. Chem. Soc. 2017, 139, 13616.

(c) Zhang, Y. G.; Ngeow, K. C.; Ying, J. J. Org. Lett. 2007, 9, 3495.

(d) Wong, Y. C.; Jayanth, T. T.; Cheng, C. H. Org. Lett. 2006, 8 , 5613.

(e) Yan, M.; Kawamata, Y.; Baran, P. S. Angew. Chem., Int. Ed. 2018, 57, 4149 .

(f) Mohle, S.; Zirbes, M.; Rodrigo, E.; Gieshoff, T.; Wiebe, A.; Waldvogel, S. R. Angew. Chem., Int. Ed. 2018, 57, 6018.

(g) Cheng, L.; Ge, X.; Liu, X.; Feng, Y. Chin. J. Org. Chem. 2020 40, 2008 (in Chinese).

(成琳，葛新，刘学民，冯云辉，有机化学, 2020, 40, 2008.)

[3] (a) Shibatomi, K.; Narayama, A.; Soga, Y.; Muto, T.; Iwasa, S. Org. Lett. 2011, 13, 2944.

(b) Ranu, B. C.; Mandal, T. J. Org. Chem. 2004, 69, 5793

[4] (a) Zhang, X.Y.; Guo, S. R. J. Sulfur Chem. 2011, 32, 23.

(b) Sindhu, K. S.; Thankachan, A. P.; Thomas, A. M.; Anilkumar, G. Tetrahedron Lett. 2016, 3, 556.

(c) Oderinde, M. S.; Frenette, M. D.; Robbins, W.; Aquila, B.; Johannes, J. W. J. Am. Chem. Soc. 2016, 138, 1760.

[5] Brindaban, C.; Ranu; Mandal, T. J. Org. Chem. 2004, 69, 5793.

[6] (a) Yan, M.; Kawamata, Y.; Baran, P. S. Angew. Chem., Int. Ed. 
2018, 57,4149 .

(b) Waldvogel, S. R.; Lips, S.; Selt, M.; Riehl, B.; Kampf, C. J. Chem. Rev. 2018, 118, 6706.

(c) Mohle, S.; Zirbes, M.; Rodrigo, E.; Gieshoff, T.; Wiebe, A.; Waldvogel, S. R. Angew. Chem., Int. Ed. 2018, 57, 6018.

(d) Zheng, L.; Tao, K.; Guo, W. Adv. Synth. Catal. 2021, 363, 62.

(e) Li, G.; Yan, Q.; Gong, X.; Dou, X.; Yang, D. ACS Sustainable Chem. Eng. 2019, 7, 14009.

(f) Peng, S.; He, W. M.; Lin, Y. W. Chin. J. Org. Chem. 2020, 40, 541 (in Chinese).

(彭莎，何为民，林英武，有机化学，2020，40, 541.)

(g) Chen, N.; Liu, B.; Liu, Y. J.; Lei, J.; Sun, K. Adv. Synth. Catal. 2020, 362, 3709.

(h) Wang, X.; Zhang, Y.; Sun, K.; Meng, J.; Zhang, B. Chin. J. Org. Chem. 2021, 41, 4588 (in Chinese).

(王薪, 张艳, 孙凯, 孟建萍, 张冰, 有机化学, 2021, 41, 4588.)

(i) Wu, Y.; Chen, J.-Y.; Ning, J.; Jiang, X.; Deng, J.; Deng, Y.; Xu, R.; He, W.-M. Green Chem. 2021, 23, 3950.

(j) Jiang, J.; Xiao, F.; He, W.-M.; Wang, L. Chin. Chem. Lett. 2021, $32,1637$.

(k) Jiang, J.; Wang, Z.; He, W.-M. Chin. Chem. Lett. 2021, 32, 1591.

(l) Mamat, M.; Liu, C.; Abdukerem D.; Abdukader, A. Org. Biomol. Chem. 2021, 19, 9855.

(m) Wang, C.; Shi, H.; Deng, G.-J.; Huang, H. Org. Biomol. Chem. 2021, 19, 9177.

[7] (a) Girard, S. A.; Knauber, T.; Li, C.-J. Angew. Chem., Int. Ed. 2014, 53,74 .

(b) Siddaraju, Y.; Prabhu, K. R. J. Org. Chem. 2016, 81, 7838.

(c) Siddaraju, Y.; Prabhu, K. R. Org. Lett. 2016, 18, 6090.

[8] Wang, P.; Tang, S.; Huang, P.; Lei, A. Angew. Chem., Int. Ed. 2017,
56,3009 .

[9] Guo, W.; Tan, W.; Zhao, M.; Tao, K.; Zheng, L. Y.; Wu, Y.; Chen, D.; Fan, X. L. RSC Adv. 2017, 7, 37739.

[10] Varun, B. V.; Gadde, K.; Prabhu, K. R. Org. Lett. 2015, 17, 2944.

[11] (a) Siddaraju, Y.; Prabhu, K. R. Org. Biomol. Chem. 2017, 15, 5191.

(b) Song, S.; Sun, X.; Li, X.; Yuan, Y.; Jiao, N. Org. Lett. 2015, 17, 2886.

(c) Rafique, J.; Saba, S.; Rosário, A. R; Braga, A. L. Chem.-Eur. J. 2016, 22, 11854 .

(d) Siddaraju, Y.; Prabhu, K. R. J. Org. Chem. 2017, 82, 3084

[12] Cao, H.; Yuan, J.; Liu, C.; Hu, X.; Lei, A. RSC Adv. 2015, 5, 41493.

[13] (a) Li, Y.; Liu, D.; Liu, C.; Lei, A. Chem.-Asian J. 2016, 11, 2246.

(b) Sumino, S.; Fusano, A.; Fukuyama, T.; Ryu, I. Acc Chem. Res. 2014, 47, 1563.

(c) Nguyen, J. D.; D'Amato, E. M.; Narayanam. J. M.; Stephenson, C. R. Nat. Chem. 2012, 4, 854.

[14] (a) Zhu, X.; Xie, X.; Li, P.; Guo, J.; Wang, L. Org. Lett. 2016, 18 , 1546.

(b) Tan, H.; Li, H. J.; Ji, W.; Wang, L. Angew. Chem., Int. Ed. 2015, 54,8374 .

(c) Yang, W.; Yang, S.; Li, P.; Wang, L. Chem. Commun. 2015, 51, 7520.

(d) Xia, D.; Miao, T.; Li, P.; Wang, L. Chem.-Asian J. 2015, 10, 1919.

[15] Chen, Q.; Wang, X. F.; Wen, C. X.; Huang, Y. L.; Yan, X. X.; Zeng, J. K. RSC Adv. 2017, 7, 39758.

[16] Tan, J.; Liang, F. S.; Wang, Y. M.; Cheng, X.; Liu, Q.; Yuan, H. Org. Lett. 2008, 10, 2485.

[17] Sun, J.; Qiu, J. K.; Zhu, Y. L.; Guo, C.; Hao, W. J.; Jiang, B.; Tu, S. J. J. Org. Chem. 2015, 80, 8217.

(Zhao, C.) 